\title{
Proportional-integral-plus control applications of state-dependent parameter models
}

\author{
C J Taylor ${ }^{1 *}$, E M Shaban ${ }^{1}$, M A Stables ${ }^{1}$, and S Ako ${ }^{2}$ \\ ${ }^{1}$ Engineering Department, Lancaster University, Lancaster, UK \\ ${ }^{2}$ Bachy Soletanche Limited, Burscough, UK
}

The manuscript was received on 10 October 2006 and was accepted after revision for publication on 23 May 2007.

DOI: 10.1243/09596518JSCE366

\begin{abstract}
This paper considers proportional-integral-plus (PIP) control of non-linear systems defined by state-dependent parameter models, with particular emphasis on three practical demonstrators: a microclimate test chamber, a 1/5th-scale laboratory representation of an intelligent excavator, and a full-scale (commercial) vibrolance system used for ground improvement on a construction site. In each case, the system is represented using a quasi-linear state-dependent parameter (SDP) model structure, in which the parameters are functionally dependent on other variables in the system. The approach yields novel SDP-PIP control algorithms with improved performance and robustness in comparison with conventional linear PIP control. In particular, the new approach better handles the large disturbances and other non-linearities typical in the application areas considered.
\end{abstract}

Keywords: control system design, non-minimal state space, state-dependent parameters, hydraulic actuators, system identification

\section{INTRODUCTION}

Previous papers have considered the proportionalintegral-plus (PIP) controller, in which non-minimal state-space (NMSS) models are formulated so that full state variable feedback control can be implemented directly from the measured input and output signals of the controlled process, without resort to the design and implementation of a deterministic state reconstructor (observer) or a stochastic Kalman filter [1-3].

Such PIP control systems have been successfully employed in a range of practical applications, particularly in the areas of microclimate control for agricultural buildings $[\mathbf{4}, \mathbf{5}]$, and in the automation of construction robots on building sites [6, 7]. In both these application areas, the most common types of controller used previously have been derived from the ubiquitous proportional-integral-derivative (PID) approach (see, for example, references [8] and [9]).

In this regard, PIP control can be interpreted as one logical extension of conventional PI/PID methods,

* Corresponding author: Engineering Department, Lancaster University, Faculty of Science and Technology, Bailrigg, Lancaster LA1 4YR, UK. email: c.taylor@lancaster.ac.uk but with additional dynamic feedback and input compensators introduced automatically when the process has second-order or higher dynamics, or pure time delays greater than unity. In contrast to conventional PI/PID control, however, PIP design exploits state variable feedback (SVF) methods, where the vagaries of manual tuning are replaced by pole assignment or linear quadratic (LQ) design.

To date, however, inherent non-linearities in the PIP system have been accounted for in a rather ad hoc manner at the design stage, sometimes leading to reduced control performance. For example, pressure disturbances sometimes take the ventilation rate in a building sufficiently far from the operating condition on which the linear controller is based for the response to such a disturbance to be relatively slow. Similarly, on a construction site, the behaviour of hydraulically driven manipulators is dominated by the highly non-linear, lightly damped dynamics of the actuators [10].

To improve PIP control in such cases, therefore, the present paper identifies, and subsequently exploits for control system design, state-dependent parameter (SDP) models. Here, the non-linear system is modelled using a quasi-linear structure in which the parameters vary as functions of the state variables, 
e.g. Young [11]. The linear-like, affine structure of the SDP model means that, at each sampling instant, it can be considered as a 'frozen' linear system. This formulation may then be used to design an SDP-PIP control law at each sampling instant using linear methods [12].

The present paper develops this new approach for three practical demonstrators, related to both the above application areas: a microclimate test chamber representing a section of a livestock building, a 1/5thscale laboratory model of an intelligent excavator, and a full-scale (commercial) vibrolance system used for ground improvement on a construction site.

With regard to the first of these examples, the paper focuses on the control of ventilation rate using an axial fan. Ventilation is one of the most significant inputs in the control of the microclimate surrounding plants or animals within the majority of agricultural buildings [13]. For example, without adequate fresh air supply within a livestock enclosure, animal comfort and welfare are drastically reduced, especially during high-density occupation, where excessive levels of moisture, heat, and internal gases are generated.

By contrast, in the civil and construction industries, semi-automatic functions are starting to be adopted as a means of improving efficiency, quality, and safety. The control problem is made difficult by a range of factors that include highly varying loads, speeds, and geometries, as well as the soil-tool interaction in the case of autonomous excavators $[\mathbf{1 4}, \mathbf{1 5}]$. In the present paper, inverse kinematics is utilized online to convert the task into a desired trajectory in the joint space, while a feedback controller regulates the applied voltage to the hydraulics, so as to maintain these joint angles.

In each case, the novel SDP-PIP approach is compared with a benchmark linear PIP design. Sections 2 and 3 of the paper describe the identification and control methodologies. Sections 4 to 6 introduce each demonstrator and present the implementation results. Finally, the conclusions are given in section 7 .

\section{SYSTEM IDENTIFICATION}

The controllers used in this paper are based on the simplest multiple-loop, single-input, single-output models. In the case of the robot arms, there is some interaction between the various joint hydraulics, but the implementation results considered below suggest that a fully multivariable control approach [4] is not required in practice.

\subsection{Model structure}

Consider the deterministic form of the SDP model $[11,16]$

$$
y_{k}=\boldsymbol{w}_{k}^{\mathrm{T}} \boldsymbol{p}_{k}
$$

where $\boldsymbol{w}_{k}^{\mathrm{T}}$ is a vector of lagged input and output variables and $\boldsymbol{p}_{k}$ is a vector of SDP parameters, defined as follows

$$
\begin{aligned}
& \boldsymbol{w}_{k}^{\mathrm{T}}=\left[\begin{array}{lllllll}
-y_{k-1} & -y_{k-2} & \cdots & -y_{k-n} & u_{k-1} & \cdots & u_{k-m}
\end{array}\right] \\
& \boldsymbol{p}_{k}=\left[\begin{array}{lllllll}
a_{1}\left\{\boldsymbol{\chi}_{k}\right\} & a_{2}\left\{\boldsymbol{\chi}_{k}\right\} & \cdots & a_{n}\left\{\boldsymbol{\chi}_{k}\right\} & b_{1}\left\{\boldsymbol{\chi}_{k}\right\} & \cdots & b_{m}\left\{\boldsymbol{\chi}_{k}\right\}
\end{array}\right]^{\mathrm{T}}
\end{aligned}
$$

Here, $y_{k}$ is the output and $u_{k}$ the control input, while $a_{i}\left\{\chi_{k}\right\} \quad(i=1,2, \ldots, n)$ and $b_{j}\left\{\chi_{k}\right\} \quad(j=1, \ldots, m)$ are state-dependent parameters. The latter are assumed to be functions of a non-minimal state vector $\chi_{k}^{\mathrm{T}}=\left[\begin{array}{ll}\boldsymbol{w}_{k}^{\mathrm{T}} & \boldsymbol{U}_{k}\end{array}\right]$ in which $\boldsymbol{U}_{k}=\left[U_{1, k}, U_{2, k}, \ldots, U_{r, k}\right]$ is a vector of other variables, not necessarily $y_{k}$ or $u_{k}$. However, for SDP-PIP control system design, it is usually sufficient to limit model (1) to the case where $\chi_{k}^{\mathrm{T}}=\boldsymbol{w}_{k}^{\mathrm{T}}$. Any pure time delay $\tau \geqslant 1$ is represented by setting the leading $b_{1}\left\{\chi_{k}\right\} \ldots b_{\tau-1}\left\{\chi_{k}\right\}$ terms to zero. Finally, $n$ and $m$ are integers representing the maximum lag associated with the output and input variables respectively.

Numerous recent publications describe an approach for the identification and estimation of the SDP model defined by equation (1), together with the application of such methods to a wide range of environmental, biological, and engineering systems (see references [11] and [16] and the references therein). In the present context, the approach is broadly composed of two distinct stages, as discussed below.

\subsection{Model identification}

The underlying model structure and potential state variables are first identified by statistical estimation of discrete-time linear transfer function models. Such models take a similar form to equation (1) but with time-invariant parameters, i.e. $a_{i}(i=1,2, \ldots, n)$ and $b_{j}(j=1, \ldots, m)$ are constant coefficients, estimated using the simplified refined instrumental variable (SRIV) algorithm [17, 18].

Two main statistical measures are utilized to help identify the most appropriate linear model structure, i.e. the values of $n$ and $m$ and the time delay $\tau$. These are the coefficient of determination $R_{\mathrm{T}}^{2}$, based on the response error, and Young's identification criterion, which provides a combined measure of model fit and parametric efficiency $[\mathbf{1 7}, \mathbf{1 8}]$.

The second stage of the analysis is based on the estimation of stochastic time-varying parameter (TVP) models using recursive Kalman filtering (KF) and fixed interval smoothing (FIS) algorithms $[\mathbf{1 9}, 20]$. 
However, since the changes in the parameters are functions of the state variables, the system may exhibit severe non-linear or even chaotic behaviour. Normally, this cannot be approximated in a simple TVP manner because the parameters can vary at a very rapid rate. For this reason, recourse is made to a novel approach that again exploits recursive KF/FIS estimation but this time within an iterative 'backfitting' algorithm that involves special reordering of the time series data $[\mathbf{1 1}, \mathbf{1 6}]$.

\subsection{Parameter estimation}

Each $a_{i}\left\{\chi_{k}\right\}$ and $b_{j}\left\{\chi_{k}\right\}$ in model (1) can be considered as a 'non-parametric' estimate because it has a different value at each sample and can only be viewed in complete form as a graph. However, it is possible to proceed to a final parametric estimation stage where the non-parametrically defined nonlinearities obtained initially by KF/FIS estimation are now parameterized in some manner in terms of their associated dependent variable.

This can be achieved by defining an appropriate parametric model in some convenient form, such as a trigonometric function, a radial basis function, or a neural network. The parameters of this parameterized model can then be estimated directly from the input-output data using some method of dynamic model optimization, e.g. deterministic nonlinear least squares or a more statistically efficient stochastic method, such as maximum likelihood.

However, for the practical applications considered below, polynomial or linear functions of the state variables are sufficient for control system design. The associated coefficients are straightforward to estimate using standard numerical optimization functions, such as fminsearch in MATLAB ${ }^{\circledR}$. In this case, the initial conditions for the optimization are based on the SDP estimates obtained from the earlier KF/FIS stage of the analysis. This helps to avoid the potential problem of fminsearch finding a local (rather than global) optimal set of parameters.

\section{CONTROL METHODOLOGY}

The NMSS representation of system (1) is

$$
\begin{aligned}
& \boldsymbol{x}_{k+1}=\mathbf{F}_{k} \boldsymbol{x}_{k}+\boldsymbol{g}_{k} u_{k}+\boldsymbol{d} y_{d, k} \\
& y_{k}=\boldsymbol{h} \boldsymbol{x}_{k}
\end{aligned}
$$

where the non-minimal state vector at the $k$ th sample is defined as

$$
\boldsymbol{x}_{k}=\left[\begin{array}{lllllll}
y_{k} & \cdots & y_{k-n+1} & u_{k-1} & \cdots & u_{k-m+1} & z_{k}
\end{array}\right]^{\mathrm{T}}
$$

and $z_{k}=z_{k-1}+\left[y_{d, k}-y_{k}\right]$ is the integral of error between the reference or command input $y_{d, k}$ and the sampled output $y_{k}$. As usual for NMSS design, inherent type 1 servomechanism performance is introduced by means of this integral-of-error state [1]. The state transition matrix $\mathbf{F}_{k}$ and input vector $\boldsymbol{g}_{k}$ at the $k$ th sample, together with the time-invariant command $\boldsymbol{d}$ and observation vectors $\boldsymbol{h}$, are defined as follows

$$
\begin{aligned}
& \mathbf{F}_{k}=\left[\begin{array}{ll}
\mathbf{F}_{1, k} & \mathbf{F}_{2, k}
\end{array}\right] \\
& \boldsymbol{g}_{k}=\left[\begin{array}{lllllllllll}
b_{1}\left\{\boldsymbol{\chi}_{k+1}\right\} & 0 & 0 & \cdots & 0 & 1 & 0 & \cdots & 0 & -b_{1}\left\{\boldsymbol{\chi}_{k+1}\right\}
\end{array}\right]^{\mathrm{T}} \\
& \boldsymbol{d}=\left[\begin{array}{llllll}
0 & 0 & 0 & \cdots & 0 & 1
\end{array}\right]^{\mathrm{T}} \\
& \boldsymbol{h}=\left[\begin{array}{llllll}
1 & 0 & 0 & \cdots & 0 & 0
\end{array}\right]
\end{aligned}
$$

with the components

$$
\mathbf{F}_{1, k}=\left[\begin{array}{ccccc}
-a_{1}\left\{\chi_{k+1}\right\} & -a_{2}\left\{\chi_{k+1}\right\} & \cdots & -a_{n-1}\left\{\chi_{k+1}\right\} & -a_{n}\left\{\chi_{k+1}\right\} \\
1 & 0 & \cdots & 0 & 0 \\
0 & 1 & \cdots & 0 & 0 \\
\vdots & \vdots & \ddots & \vdots & \vdots \\
0 & 0 & \cdots & 1 & 0 \\
0 & 0 & \cdots & 0 & 0 \\
0 & 0 & \cdots & 0 & 0 \\
\vdots & \vdots & \ddots & \vdots & \vdots \\
0 & 0 & \cdots & 0 & 0 \\
a_{1}\left\{\chi_{k+1}\right\} & a_{2}\left\{\chi_{k+1}\right\} & \cdots & a_{n-1}\left\{\chi_{k+1}\right\} & a_{n}\left\{\chi_{k+1}\right\}
\end{array}\right]
$$




$$
\mathbf{F}_{2, k}=\left[\begin{array}{ccccc}
b_{2}\left\{\chi_{k+1}\right\} & \cdots & b_{m-1}\left\{\chi_{k+1}\right\} & b_{m}\left\{\chi_{k+1}\right\} & 0 \\
0 & 0 & 0 & 0 & 0 \\
0 & 0 & 0 & 0 & 0 \\
\vdots & \vdots & \vdots & \vdots & 0 \\
0 & \cdots & 0 & 0 & 0 \\
0 & \cdots & 0 & 0 & 0 \\
1 & \cdots & 0 & 0 & 0 \\
\vdots & \ddots & \vdots & \vdots & \vdots \\
0 & \cdots & 1 & 0 & 0 \\
-b_{2}\left\{\chi_{k+1}\right\} & \cdots & -b_{m-1}\left\{\chi_{k+1}\right\} & -b_{m}\left\{\chi_{k+1}\right\} & 1
\end{array}\right]
$$

Note that the elements of the $(n+1)$ th row of $\mathbf{F}_{k}$ are all zero if $m>1$.

\subsection{Control algorithm}

The control law takes the usual SVF form

$$
v_{k}=-\boldsymbol{l}_{k} \boldsymbol{x}_{k}
$$

where the control gain vector

$$
\boldsymbol{l}_{k}=\left[\begin{array}{lllllll}
f_{0, k} & \cdots & f_{n-1, k} & g_{1, k} & \cdots & g_{m-1, k} & -k_{\mathrm{I}, k}
\end{array}\right\}
$$

is obtained at each sampling instant by either pole assignment or optimization of an LQ cost function. With regard to the latter approach, earlier research has either used a 'frozen-parameter' system defined as a sample member of the family of NMSS models $\left\{\mathbf{F}_{k}, \boldsymbol{g}_{k}, \boldsymbol{d}, \boldsymbol{h}\right\}$ or has solved the discrete-time algebraic Riccatti equation at each sampling instant [12]. For pole assignment, the control gains are similarly determined using linear methods. One approach, for example, involves definition of a generic $\boldsymbol{\Sigma}$ matrix [1] Alternatively, it is straightforward to derive algebraic solutions for specific cases, as illustrated in reference [21].

Note that the expected design response in the pole assignment case, such as dead-beat or a specified degree of overshoot, is only obtained when the most recent SDP estimates are utilized, i.e. $\mathbf{F}_{k}$ and $\boldsymbol{g}_{k}$ are defined in terms of $\chi_{k+1}$ rather than $\chi_{k}$ in equations (2). This result mirrors that found for PIP control of bilinear systems, a special case of the SDP model considered here [22]. In a similar manner to the present example, reference [23] demonstrates that the bilinear parameter utilized in the control law should be based on $k+1$ rather than $k$.

Figure 1 illustrates the SDP-PIP controller in block diagram form, where the control polynomials are given by $F_{1, k}\left(\mathrm{z}^{-1}\right)=f_{1, k}+\cdots+f_{n-1, k}$ and $G_{k}\left(\mathrm{z}^{-1}\right)=$ $1+g_{1, k}+\cdots+g_{m-1, k}$, while $f_{0, k}$ and $k_{1, k}$ are the proportional and integral gains respectively. Finally,

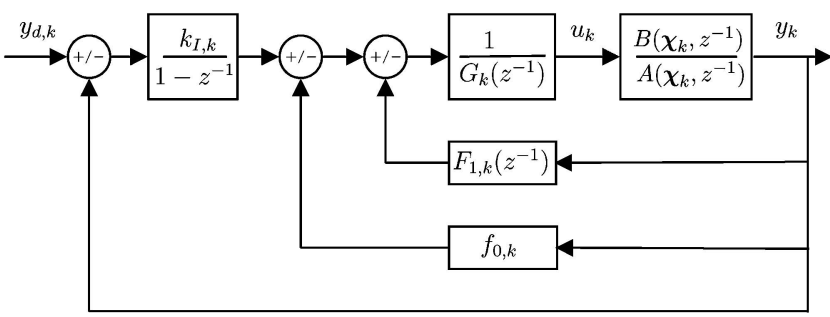

Fig. 1 SDP-PIP control implemented in feedback form

$A\left(\chi_{k}, \mathrm{z}^{-1}\right)$ and $B\left(\chi_{k}, \mathrm{z}^{-1}\right)$ in Fig. 1 represent the SDP model (1) in polynomial form, similar to a transfer function but with state-dependent parameters $[11,16]$.

\subsection{Controllability}

A prerequisite of global controllability is that the system $\left\{\mathbf{F}_{k}, \boldsymbol{g}_{k}, \boldsymbol{h}, \boldsymbol{d}\right\}$ is piecewise controllable at each sample $k$, with the standard NMSS controllability conditions applying over the sampling period. This requirement follows from the fact that, if a system is globally controllable, it clearly has to be locally controllable. Although omitted here for brevity, such NMSS/PIP linear controllability conditions are derived by Young et al. [1].

The first condition states that there should be no pole zero cancellations in the model, while the second avoids the presence of a zero at unity, which would cancel with the unity pole associated with the integral action. In the SDP-PIP case, it is straightforward to check these conditions at every sampling instant to ensure local controllability. If they fail to hold at the $k$ th sample, model (1) is instead evaluated for $k-1$. Unfortunately, this effectively leaves the system in linear (fixed-gain) mode for a period of time, something that can be undesirable in practice.

For this reason, the present research first identifies any problem regions of the parameter space by offline simulation. This is feasible because, in practice, the system variables will always be constrained to lie within a certain range. For example, the boom angle of the vibrolance system is limited by hardware to $0 \rightarrow 60^{\circ}$, while the input is a voltage signal scaled within \pm 1000 (section 6). The trajectory of the command input is subsequently chosen to avoid local controllability problems, if any arise. Of course, such an approach is necessarily based on a caseby-case empirical study and, as the order of the system increases (for example with multiple state dependencies), requires an increasing computational burden to evaluate [24].

Although derivation of global controllability results for the non-linear SDP-PIP system is the subject 
of ongoing research by the authors, the pragmatic approach described above appears satisfactory for all three applications considered in the present paper, as shown below.

\section{VENTILATION CHAMBER}

Taylor [25] describes the utility of a $2 \mathrm{~m}^{2}$ by $1 \mathrm{~m}$ forced-ventilation test chamber at Lancaster University. A computer-controlled axial fan is positioned at the outlet in order to draw air through the chamber, while an air velocity transducer measures the associated ventilation. The inlet airflow is independently regulated by a second fan, utilized to represent realistic pressure disturbances and external wind conditions.

\subsection{SDP model for ventilation rate}

Analysis of experimental data from the chamber yields the following SDP model for ventilation rate, with $\tau=2$ sample time delays [21]

$$
\begin{aligned}
& y_{k}=-a_{1}\left\{\chi_{k}\right\} y_{k-1}+b_{2}\left\{\chi_{k}\right\} u_{k-2} \\
& a_{1}\left\{\chi_{k}\right\}=\alpha_{1}+\alpha_{2} y_{k-1} \\
& b_{2}\left\{\chi_{k}\right\}=\frac{\phi\left\{u_{k-2}\right\}\left(1+a_{1}\left\{y_{k-1}\right\}\right)}{u_{k-2}}
\end{aligned}
$$

where $\alpha_{1}$ and $\alpha_{2}$ are constant coefficients, $\phi\left\{u_{k-2}\right\}$ is the non-linear relationship discussed below, $y_{k}$ is the outlet air velocity $(\mathrm{m} / \mathrm{s})$, and $u_{k}$ is the voltage applied to the fan, expressed as a percentage of the maximum voltage. Equation (4) is based on a sampling rate of $2 \mathrm{~s}$, which yields a good compromise between a fast response and a desirable low-order model.

At high applied voltages, the steady state airflow rate converges asymptotically to a maximum value determined by the characteristics of the fan, defined by the following relationship based on a logistic growth function

$$
y_{\infty}=\phi\left\{u_{\infty}\right\}=\left\{\frac{y_{\max }}{1+\mathrm{e}^{-\theta\left(u_{\infty}-x_{0}\right)^{1 / c}}}\right\}
$$

where $y_{\infty}$ is the steady-state air velocity, $u_{\infty}$ is a constant applied voltage, and $y_{\max }, \theta, x_{0}$, and $c$ are coefficients. Figure 2 illustrates the estimated linear state dependency for $a_{1}\left\{\chi_{k}\right\}$ and the power curve equation (5).

\subsection{Implementation}

For the present example, SDP-PIP design is based on pole positions of 0.7 on the complex $z$ plane.
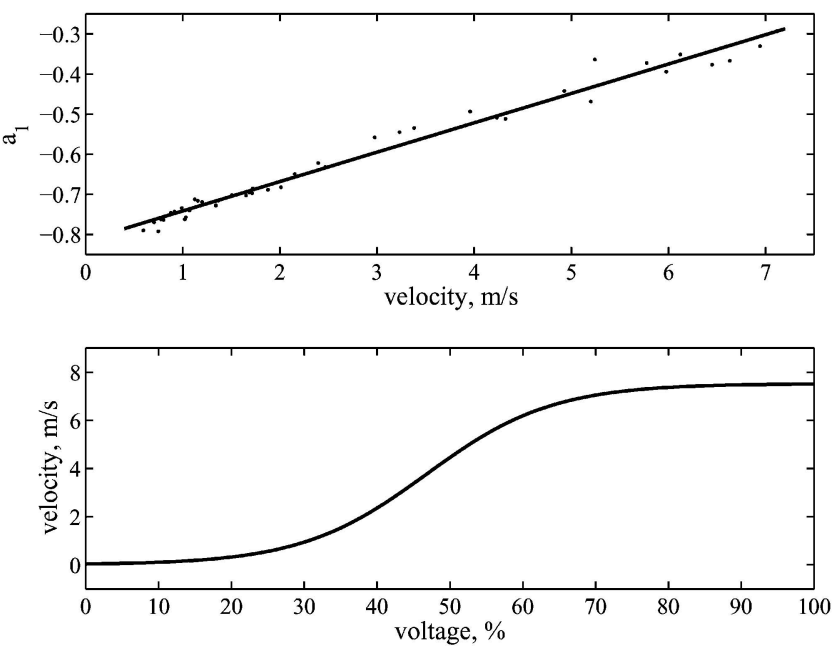

Fig. 2 Ventilation rate SDP model. Upper graph: $a_{1}\left\{\chi_{k}\right\}$ estimates (dots) and least-squares fitted straight line (solid trace) plotted against air velocity $(\mathrm{m} / \mathrm{s})$. Lower graph: power curve represented by equation (5), showing the steady-state air velocity $(\mathrm{m} / \mathrm{s})$ plotted against the scaled voltage input $(\%)$

For comparison, a fixed-gain PIP controller is also developed for an operating level of $4-5 \mathrm{~m} / \mathrm{s}$. As would be expected, the performance of the SDP-PIP and linear PIP designs are very similar for ventilation rates close to this operating level.

By contrast, Fig. 3 shows the advantage of the non-linear approach when low airflow rates are encountered (similar results emerge for high airflows).

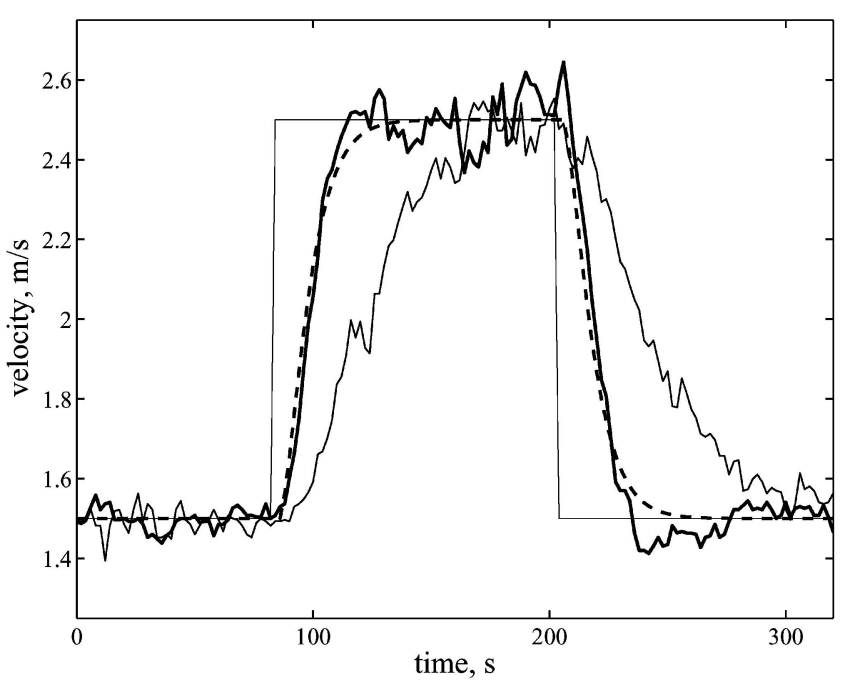

Fig. 3 Ventilation chamber implementation experiment showing the air velocity plotted against time (s): command input (step changes), SDP-PIP (thick trace), fixed-gain PIP (thin), and simulated design response (dashed) 
Figure 3 shows that the SDP-PIP-controlled output approximates the desired speed of response specified by the pole positions, determined by simulating the design polynomial in open loop. By contrast, the inherent model mismatch of the linear controller yields a significantly slower response.

In the context of agricultural buildings, the disturbance response illustrated by Fig. 4 is of particular significance. Here, the secondary fan at the chamber inlet is employed to simulate large pressure disturbances. These disturbances temporarily take the ventilation rate away from the operating level of the fixed-gain controller, and hence the SDP-PIP design reacts fastest to the problem.
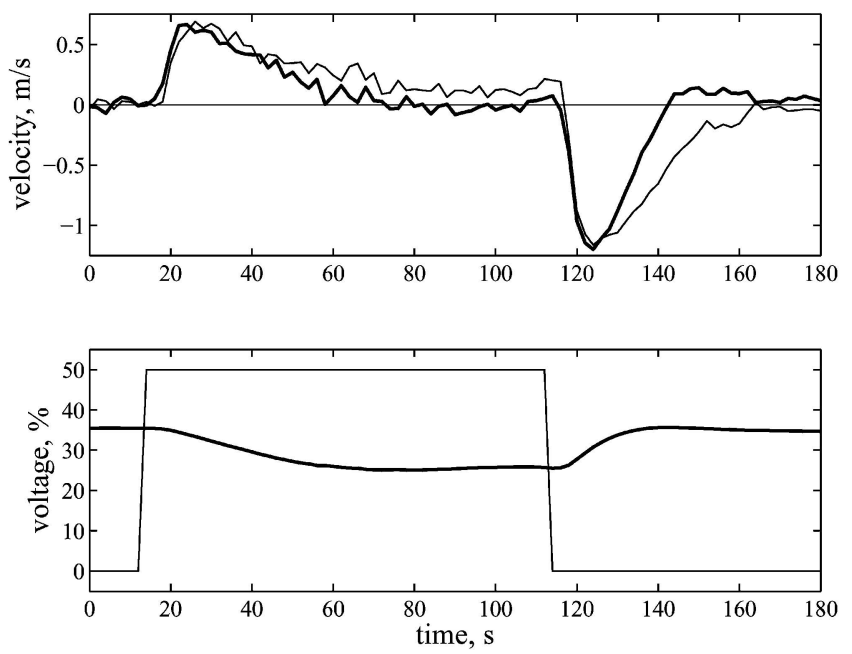

Fig. 4 Ventilation chamber disturbance experiment. Upper graph: air velocity with the operating level removed, plotted against time (s): command input (constant), SDP-PIP (thick trace), and fixed-gain PIP (thin). Lower graph: SDP-PIP control input (thick) and disturbance fan input (thin), both scaled voltages (\%)

\section{LABORATORY EXCAVATOR}

The laboratory robot arm is a $1 / 5$ th-scale representation of the more widely known Lancaster University computerized intelligent excavator (LUCIE), which has been developed to dig trenches on a construction site $[6,9]$. It provides a valuable test bed for the safe development of new control strategies before implementation on full-scale systems.

As illustrated in Fig. 5, the arm consists of four hydraulically actuated joints. The joint angles are measured directly by mounting rotary potentiometers concentrically with each joint pivot. These signals are routed to high-linearity instrumentation amplifiers within the card rack for conditioning before forwarding to the $A / D$ converter. The rig is supported by multiple I/O asynchronous real-time control systems, which allow for multitasking processes via modularization of code written in Turbo $\mathrm{C}++{ }^{\circledR}$.

Valve calibration is based on normalizing the input voltage of each joint into input demands, which range from -1000 for the highest possible downward velocity to +1000 for the highest possible upward velocity. Here, an input demand of zero corresponds to no movement. Note that, without such valve calibration, the arm will gradually slack down because of the payload carried by each joint (see reference [24] for details).

In open-loop mode, the arm is manually driven to dig a trench in the sandpit, with the operator using two analogue joysticks, each with two degrees of freedom. The first joystick is used to drive the boom and slew joints, while the other is used to move the dipper and bucket joints. In this manner, a skilful operator moves the four joints simultaneously to perform the task. By contrast, the objective here is to design a computer-controlled system for automatic digging without human intervention.

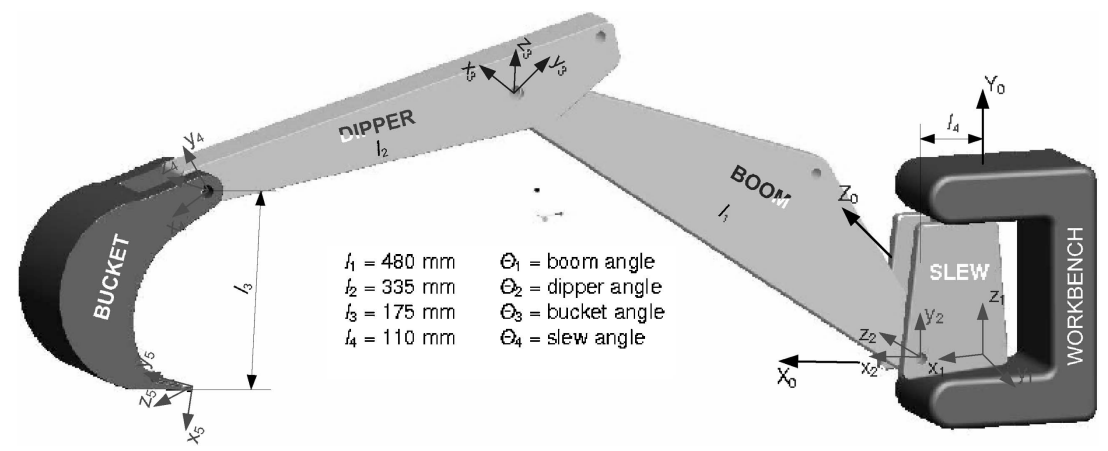

Fig. 5 Schematic diagram of the laboratory excavator, showing the link lengths and coordinate definitions for each controlled joint 


\subsection{Kinematics}

The kinematic equations below allow the tool tip to be programmed to follow the planned trajectory, while the bucket angle is separately adjusted to collect or release sand. In this regard, Fig. 5 shows the laboratory excavator and its dimensions, i.e. $\theta_{i}$ (joint angles) and $l_{i}$ (link lengths), where $i=1,2,3$, and 4 for the boom, dipper, bucket, and slew respectively.

Given $\{X, Y, Z\}$ from the trajectory planning routine, i.e. the position of the end-effector using a coordinate system originating at the workbench, together with the orientation of the bucket $\Theta=\theta_{1}+\theta_{2}+\theta_{3}$, the following inverse kinematic algorithm is derived by Shaban [24] using the well-known DenavitHartenberg convention. Here, $C_{i}$ and $S_{i}$ denote $\cos \left(\theta_{i}\right)$ and $\sin \left(\theta_{i}\right)$ respectively, while $C_{123}=\cos \left(\theta_{1}+\theta_{2}+\theta_{3}\right)$

$$
\begin{aligned}
& \theta_{4}=-\arctan \left[\frac{Z}{X}\right] \\
& \bar{X}=\frac{X-l_{4} C_{4}}{C_{4}}-l_{3} C_{123} \\
& \bar{Y}=Y-l_{3} S_{123} \\
& \theta_{1}=\arctan \left[\frac{\left(l_{1}+l_{2} C_{2}\right) \bar{Y}-l_{2} S_{2} \bar{X}}{\left(l_{1}+l_{2} C_{2}\right) \bar{X}-l_{2} S_{2} \bar{Y}}\right] \\
& \theta_{2}= \pm \arccos \left[\frac{\bar{X}^{2}+\bar{Y}^{2}-l_{1}^{2}-l_{2}^{2}}{2 l_{1} l_{2}}\right] \\
& \theta_{3}=\Theta-\theta_{1}-\theta_{2}
\end{aligned}
$$

During the excavation of a trench, each cycle can be divided into four distinct stages: positioning the bucket to penetrate the soil; the digging process in a horizontal straight line along the specified void length; picking up the collected sand from the void to the discharge side; and discharging the sand. For the present example, the speed profile typically ramps up at a constant acceleration before proceeding at a constant speed and finally ramping down to zero at a constant deceleration [24].

\subsection{SDP models for joint angle}

For linear PIP design, open-loop step experiments are first conducted for a range of applied voltages and initial conditions, all based on a sampling rate of $0.11 \mathrm{~s}$. In this case, the SRIV algorithm suggests that a first-order linear model with $\tau$ samples time delay, i.e. $y_{k}=a_{1} y_{k-1}+b_{\tau} u_{k-\tau}$ provides an approximate representation of each joint, with $\tau=1$ for the dipper and bucket joints and $\tau=2$ for the boom and slew.

Here, $y_{k}$ is the joint angle and $u_{k}$ is the scaled voltage in the range \pm 1000 , while $\left\{a_{1}, b_{\tau}\right\}$ are time- invariant parameters. Note that the arm essentially acts as an integrator, since the normalized voltage has been calibrated so that there is no movement when $u_{k}=0$. In fact, $a_{1}=-1$ is fixed a priori in the final model, so that only the numerator parameter $b_{\tau}$ is estimated in practice for linear PIP design.

However, further analysis of open-loop data reveals limitations in the linear model. In particular, the value of $b_{\tau}$ changes by a factor of ten or more, depending on the applied voltage used in the step experiments. In fact, SDP analysis suggests that a more appropriate model for the boom takes the form of equation (4) with

$$
\begin{aligned}
& a_{1}\left\{\chi_{k}\right\}=0.28 \times 10^{-6} u_{k-2}^{2}-1 \\
& b_{2}\left\{\chi_{k}\right\}=-5.8 \times 10^{-6} u_{k-2}+0.0194
\end{aligned}
$$

In this regard, Fig. 6 shows the SDP model response compared with typical open-loop data for the boom. Here, the input sequence consists of a random series of step inputs at random levels, so as to fully excite the non-linearities in the system. For this reason, only the SDP model captures the dynamic behaviour of the system $\left(R_{\mathrm{T}}^{2}=0.9\right)$, while the SRIV estimated linear model fails to converge to a useful solution $\left(R_{\mathrm{T}}^{2}<0\right)$.

Finally, Fig. 7 illustrates the parameter estimates associated with equation (12). The unsorted time histories of each SDP are shown on the left-hand subplots, while the right-hand subplots illustrate the more meaningful state dependencies. For brevity, similar SDP models for the dipper, slew, and bucket angles are omitted here. However, full details of these models and associated SDP-PIP control algorithms are reported by Shaban [24]. In this case, the control algorithms are updated at each sampling instant using linear LQ methods.

\subsection{Implementation}

Typical implementation results for the boom arm are illustrated in Fig. 8, where it is clear that the SDP-PIP algorithm is more robust than the linear PIP algorithm to large steps in the command level. In fact, the linear design yields unwelcome highfrequency oscillations in the control input signal. Note that the two controllers are designed to yield a similar speed of response to the theoretical case, shown as the dashed trace, and hence the differences seen in Fig. 8 are entirely due to the inherent nonlinearities in the system. The time-varying SDP-PIP control gains for this experiment are compared with the linear gains in Fig. 9. 

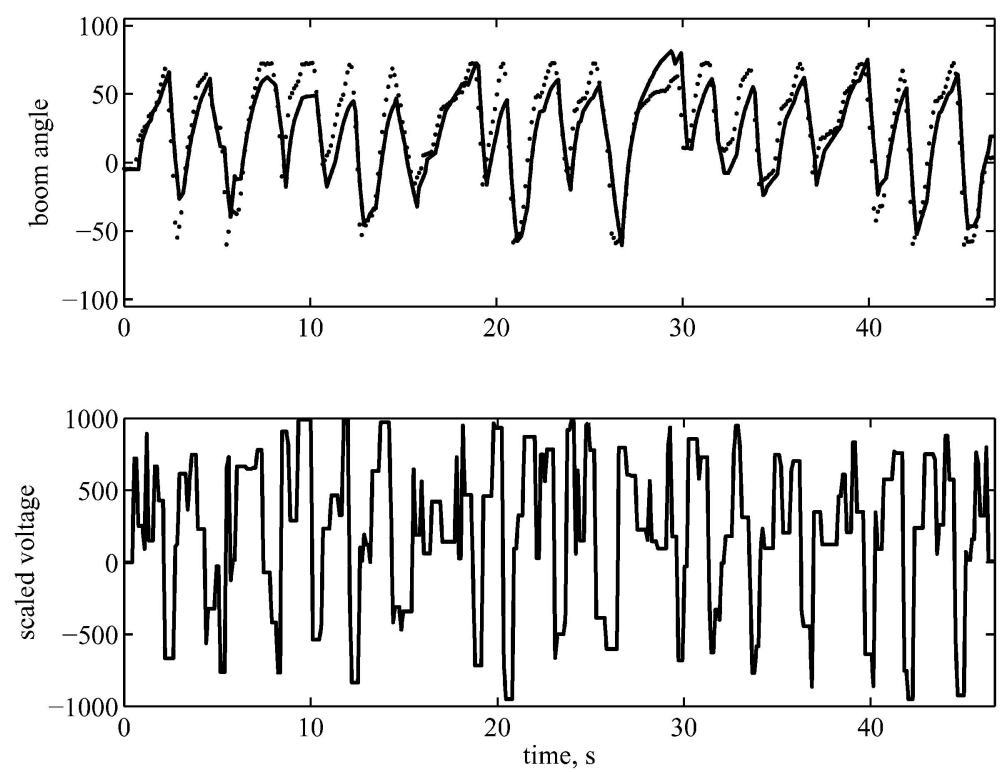

Fig. 6 Open-loop data for the laboratory excavator boom arm. Upper graph: boom angle (deg, dots) and SDP model response (solid) plotted against time (s). Lower graph: scaled input voltage
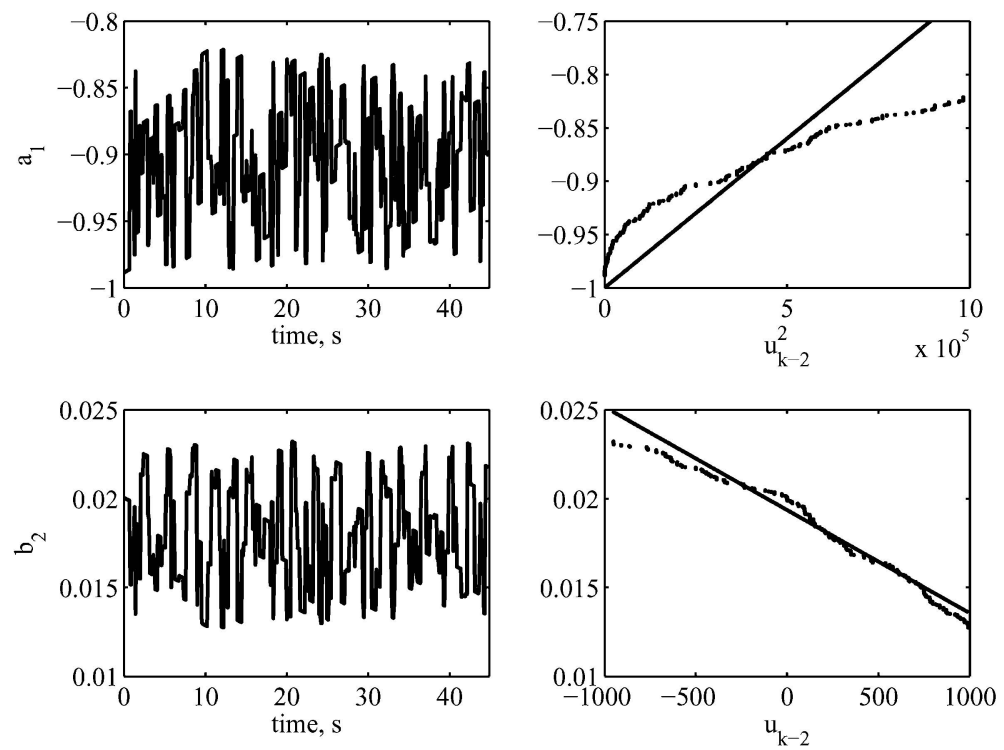

Fig. 7 Parameter estimates for the laboratory excavator boom arm. Left-hand graphs: SDP estimates plotted against time (s). Right-hand graphs: SDP estimates plotted against state variable, showing a typical realization from one experiment (dots) and the optimized fit from four datasets (solid)

It should be pointed out that the response time for this example has been deliberately increased to the practical limit of stable linear PIP control in order to emphasize these differences. By contrast, Fig. 10 illustrates control of the dipper arm using slower LQ weightings. Here, the SDP-PIP response closely follows the theoretical design response, while the linear controller is rather slower. Although the differences between the two approaches are reduced in this case, even small variations in the response time are multiplied up when the bucket position is finally resolved in the sandpit.

In the latter regard, Table 1 compares the response time of the PIP and SDP-PIP approaches, represented by the number of seconds taken to complete three complete trenches, each consisting of nine digging cycles. Here, the improved joint angle control allows for a faster SDP-PIP design, typically yielding a 10 

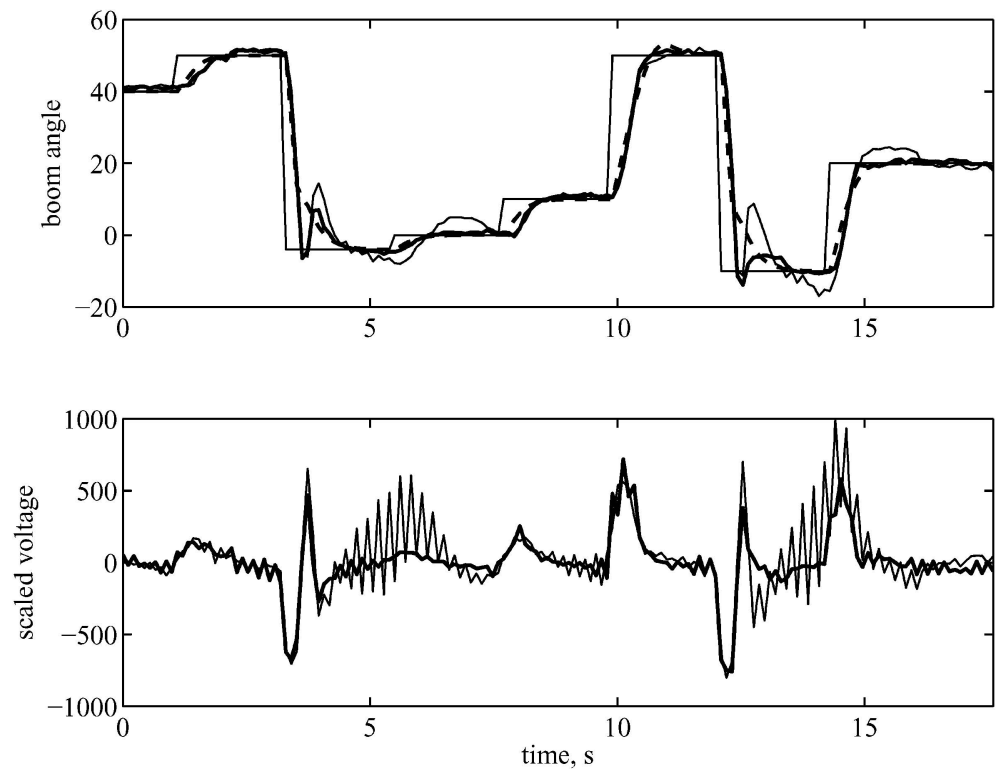

Fig. 8 Isolated control of the laboratory excavator boom arm, using relatively 'fast' control weights. Upper graph: command input (deg, sequence of step changes), theoretical response (dashed), non-linear SDP-PIP (thick), and linear PIP (thin), plotted against time (s). Lower graph: scaled input voltages
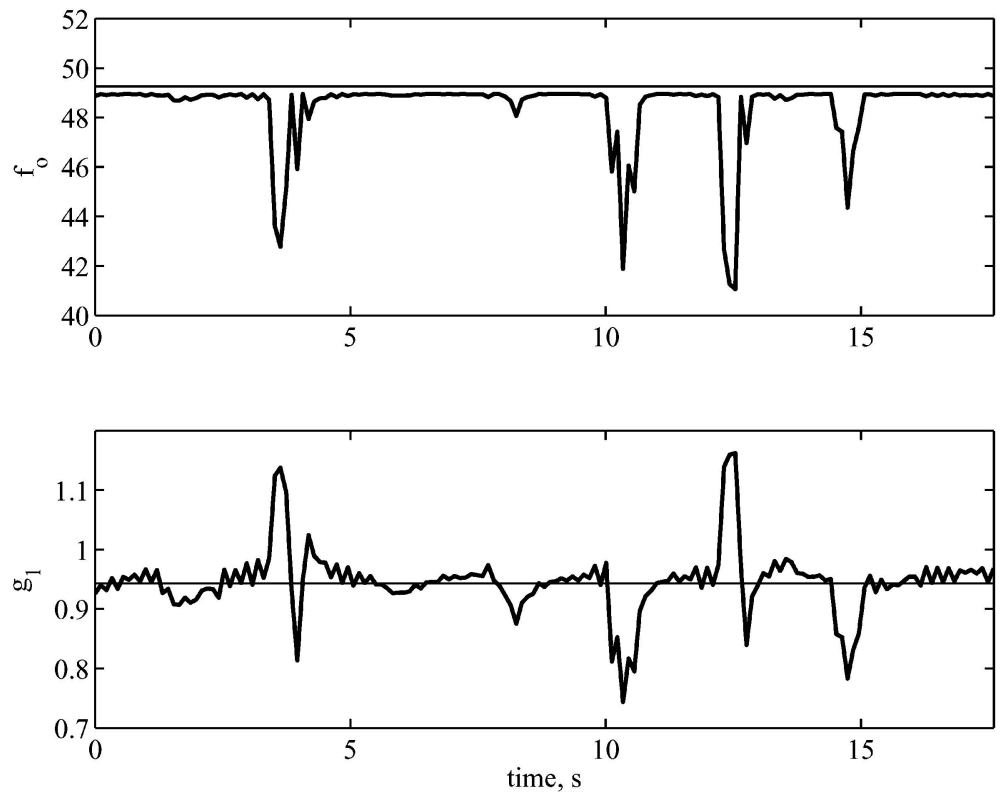

Fig. 9 Non-linear SDP-PIP (thick) and linear PIP (thin) control gains for the experiment shown in Fig. 8, plotted against time (s). Upper graph: proportional gain. Lower graph: input gain

Table 1 Time (s) taken to complete one trench

\begin{tabular}{lll}
\hline Trench & SDP-PIP & Linear PIP \\
\hline 1 & 339 & 369 \\
2 & 334 & 370 \\
3 & 336 & 373 \\
\hline
\end{tabular}

per cent reduction in the digging time. Finally, Fig. 11 illustrates typical SDP-PIP implementation results for one cycle of the bucket, showing a three-dimensional coordinate plot of the end-effector. This graph shows the bucket being first lowered into and subsequently being dragged through the sand, followed by extraction, displacement, and release. 

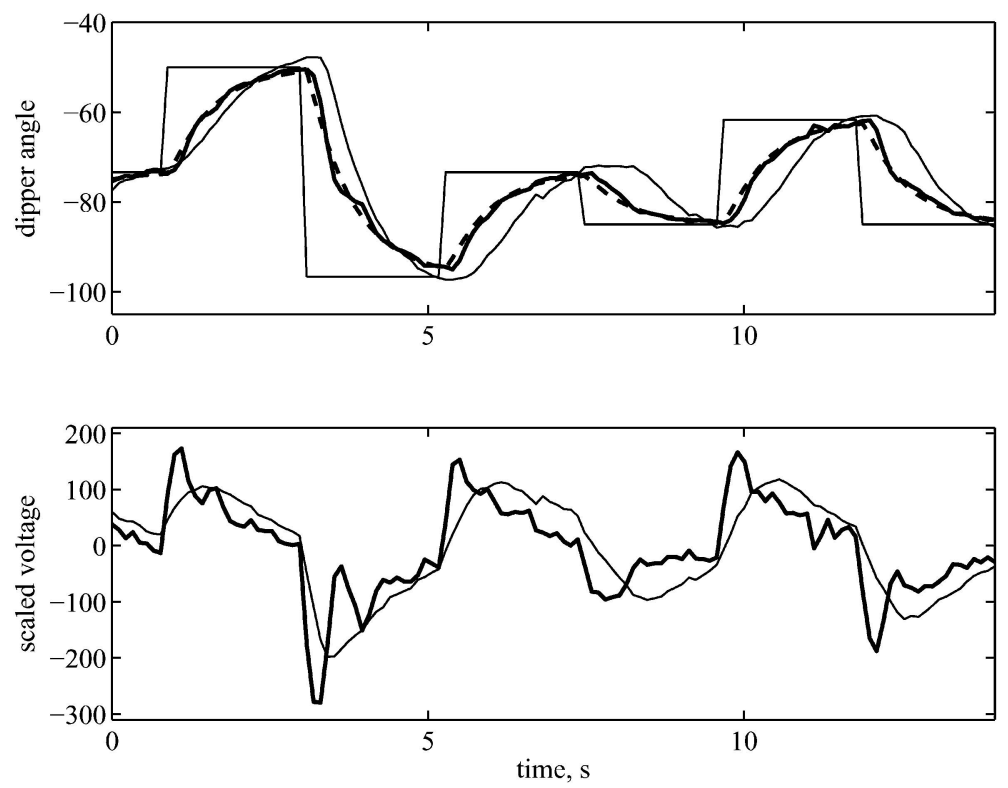

Fig. 10 Isolated control of the laboratory excavator dipper arm. Upper graph: command input (deg, sequence of step changes), theoretical response (dashed), non-linear SDP-PIP (thick), and linear PIP (thin), plotted against time (s). Lower graph: scaled input voltages

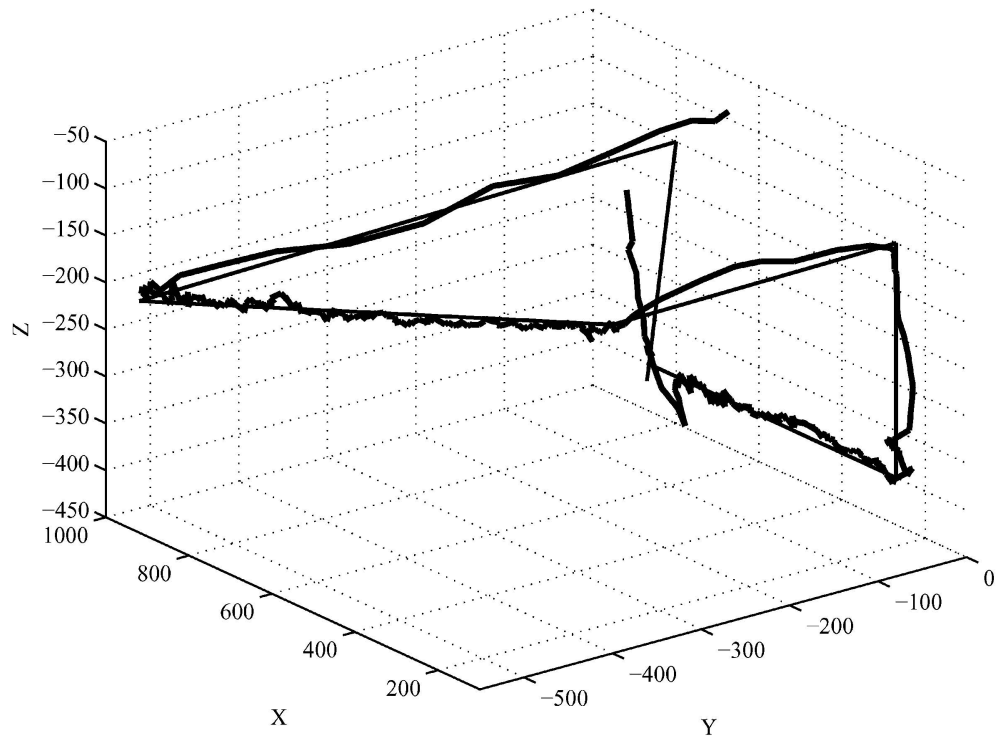

Fig. 11 Resolved position of the laboratory excavator end-effector for one digging cycle in sand using SDP-PIP control, showing the planar horizontal and vertical displacements ( $X$ and $Z$ ), together with the slew $Y$, with the setpoint shown as straight lines (mm)

\section{GROUND COMPACTION}

With regard to the full-scale commercial system, the field tests utilized a KOMATSU-PC-240-LC-7 hydraulic excavator, as illustrated in Fig. 12. The vibrolance is connected to the excavator arm and hangs freely like a pendulum (joint 4). The operator first positions the vehicle by manually adjusting the slew (joint 1 ) and excavator tracks. The vibrating probe is subsequently lowered into the ground, penetrating downwards by regulating the boom (joint 2) and dipper (joint 3).

Here, the objective is to keep the arm tip moving in a vertical straight-line path. In this manner, the surrounding soil is compacted up to a distance of about $5 \mathrm{~m}$ from the probe; for granular soils, the cavity produced is subsequently filled with gravel. Automatic control of joints 2 and 3 would provide a number of benefits, such as a reduced dependence 


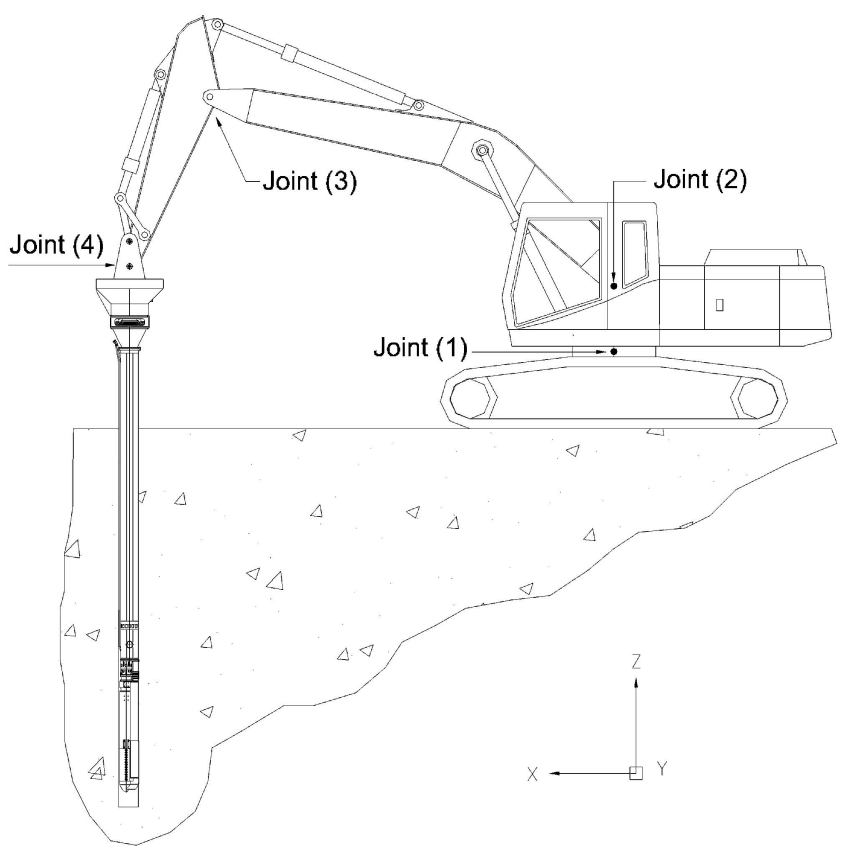

Fig. 12 Schematic diagram of the two-arm manipulator with vibrolance

on operator skills and a lower work load, both of which might be expected to contribute to improvements in quality and productivity, and an increase in tool life.

In fact, verticality errors in the uncontrolled system, particularly when the probe is raised from the soil, have previously led to probe repair costs of over $£ 8000$ on each occasion. In this regard, the present authors have recently developed a semi-automatic system, whereby the operator only moves a joystick either forwards or backwards in order to raise or lower the probe $[\mathbf{2 4}, \mathbf{2 6}]$. For brevity, the necessary kinematic equations and rule-based algorithms for determining the appropriate tool-tip trajectory are omitted here. However, in both instances, a similar approach to that of the laboratory excavator is followed (see reference [24] for details).

\subsection{SDP models for the vibrolance}

For this application, experimentation suggests that a sampling rate of $0.44 \mathrm{~s}$ provides a good compromise between a fast response and a desirable low-order model. In this case, the linear model $y_{k}=a_{1} y_{k-1}+b_{2} u_{k-2}$ again provides a reasonable explanation of the data for a wide range of operating conditions. In fact, this model has been successfully used in the design of a preliminary linear PIP control system [26]. For non-linear design, the SDP model of the boom takes the form of equation (4) with time-invariant $a_{1}\left\{\chi_{k}\right\}=a_{1}=-1$ and $b_{2}\left\{\chi_{k}\right\}$ defined as follows

$$
\begin{aligned}
b_{\mathrm{b}}\left\{\chi_{k}\right\}= & 1.5 \times 10^{-11} u_{k-2}^{3}+1.3 \times 10^{-9} u_{k-2}^{2} \\
& -1.88 \times 10^{-5} u_{k-2}+0.007
\end{aligned}
$$

The equivalent model for the dipper has

$$
b_{\mathrm{d}}\left\{\chi_{k}\right\}=-1.078 \times 10^{-5}\left|u_{k-2}\right|+0.015
$$

where $\left|u_{k-2}\right|$ represents the absolute value of the lagged input signal. In a similar manner to the laboratory excavator, $u_{k}$ is scaled in the range \pm 1000 .

\subsection{Implementation}

Solution of the discrete-time algebraic Riccatti equation for $\left\{\mathbf{F}_{k}, \boldsymbol{g}_{k}, \boldsymbol{d}, \boldsymbol{h}\right\}$, yields the following SDP-PIP gain vector for the boom angle [24]

$$
\boldsymbol{I}_{k}^{\mathrm{T}}=\left[\begin{array}{c}
22.1 \\
22.1 \times b_{\mathrm{b}}\left\{\boldsymbol{\chi}_{k}\right\} \\
-1.46
\end{array}\right]
$$

The equivalent gain vector for the dipper angle is as follows

$$
\boldsymbol{l}_{k}^{\mathrm{T}}=\left[\begin{array}{c}
25.8 \\
25.8 \times b_{\mathrm{d}}\left\{\boldsymbol{\chi}_{k}\right\} \\
-3.15
\end{array}\right]
$$

Typical closed-loop results for lowering the probe in the air are illustrated in Fig. 13. Here, it is clear that the SDP-PIP approach yields more accurate control than the linear PIP algorithm, particularly in the initial positioning of the arm. In general, SDP-PIP control appears to offer smoother, more accurate movement of the excavator tool and hence potentially allows for faster response times. Note that the slew is fixed once the probe starts to be lowered, and hence Fig. 13 shows only the $\{X, Z\}$ coordinates, while $Y$ remains constant during probe operations.

On-site implementation experiments for lowering the probe into soil using the linear PIP controller are very promising, as reported in reference [26]. Here, the error between the measured tool-tip trajectory and the horizontal setpoint is typically less than $10 \mathrm{~cm}$ for over 90 per cent of the time. To the authors' knowledge, this level of performance is not normally achieved by a skilled human operator. Furthermore, the automatic system completes an entire cycle at least as fast as a skilled human operator.

However, relatively large transient deviations from the setpoint do occasionally occur using linear PIP methods. Although these are often associated with difficult obstructions in the soil, they provide the motivation for the present research using SDP-PIP 


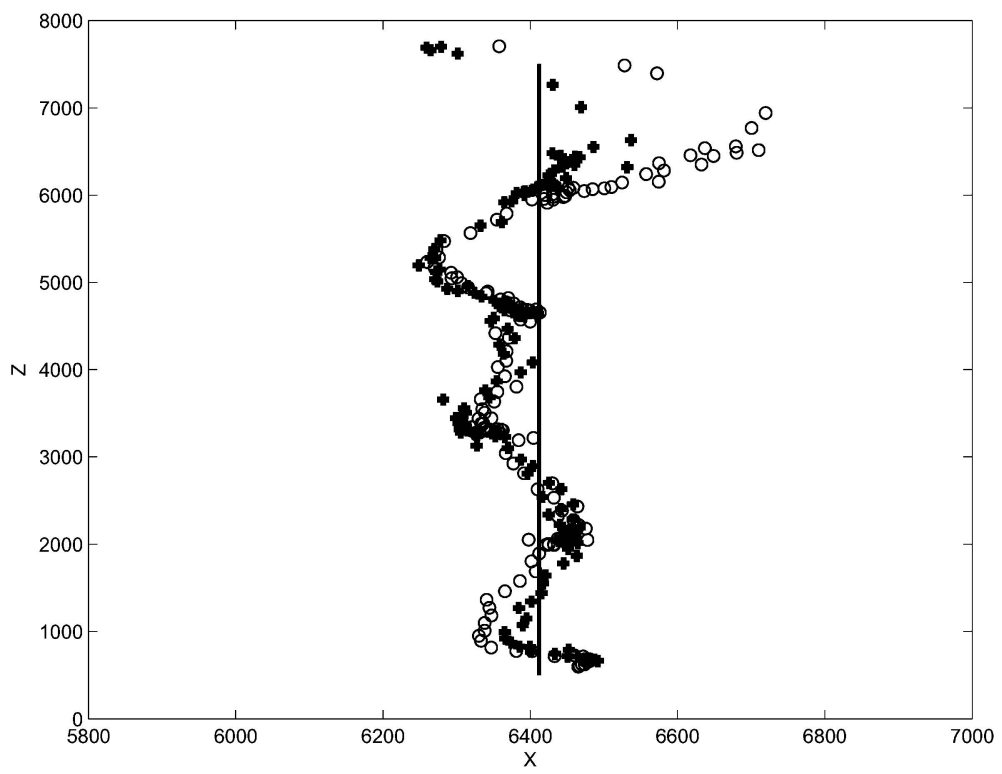

Fig. 13 Implementation experiment for the vibrolance, showing the resolved position of the end-effector, represented by the horizontal and vertical displacements ( $\mathrm{mm})$. Non-linear SDP-PIP control (crosses) is compared with linear PIP (circles) and the horizontal setpoint (solid)

methods. In this regard, Fig. 13 presages potential improvements, and it is clear that the next step of the research is to evaluate the on-site performance of the new approach. Although the commercial system used above is presently unavailable for research, the authors hope that the present paper will stimulate further interest in this area.

\section{CONCLUSIONS}

This paper has developed SDP-PIP control systems for three practical demonstrators: a forced-ventilation test chamber, a 1/5th-scale laboratory representation of an intelligent excavator, and a vibrolance system used for ground improvement on a construction site. These examples represent the first practical implementations of the recently proposed SDP-PIP control methodology.

In each case, the system was modelled using the quasi-linear SDP model structure, in which the parameters are functionally dependent on other variables in the system. This formulation is subsequently used to design an SDP-PIP control law using linear system design strategies, such as suboptimal LQ design, in which the control gains are themselves state dependent.

Practical results for the ventilation chamber reveal improved control performance in comparison with conventional PIP methods, particularly with regards to the disturbance response. In the case of the laboratory excavator, the new approach yields improved control of the joint angles and hence a reduced time to complete a trench. Finally, the vibrolance experiments similarly suggest improved performance and robustness in comparison with the linear PIP methods previously developed.

\section{ACKNOWLEDGEMENTS}

The authors are grateful for the support of the Engineering and Physical Sciences Research Council (EPSRC). Video footage of the demonstrators may be downloaded from www.lancs.ac.uk/staff/taylorcj/ nonlinear. The statistical tools and associated estimation algorithms have been assembled as the CAPTAIN toolbox [16] within the MATLAB ${ }^{\circledR}$ software environment and may be downloaded from www.es.lancs.ac.uk/cres/captain.

\section{REFERENCES}

1 Young, P. C., Behzadi, M. A., Wang, C. L., and Chotai, A. Direct digital and adaptive control by input-output, state variable feedback pole assignment. Int. J. Control, 1987, 46, 1867-1881.

2 Taylor, C. J., Chotai, A., and Young, P. C. Proportional-Integral-Plus (PIP) control of time delay systems. Proc. Instn Mech. Engrs, Part I: J. Systems and Control Engineering, 1998, 212, 37-48. 
3 Taylor, C. J., Young, P. C., and Chotai, A. State space control system design based on non-minimal state-variable feedback: further generalisation and unification results. Int. J. Control, 2000, 73, 1329-1345.

4 Young, P. C., Lees, M., Chotai, A., Tych, W., and Chalabi, Z. S. Modelling and PIP control of a glasshouse micro-climate. Control Engng Practice, 1994, 2(4), 591-604.

5 Taylor, C. J., Leigh, P. A., Chotai, A., Young, P. C., Vranken, E., and Berckmans, D. Cost effective combined axial fan and throttling valve control of ventilation rate. IEE Proc. Control Theory and Applic., 2004, 151(5), 577-584.

6 Gu, J., Taylor, C. J., and Seward, D. W. The automation of bucket position for the intelligent excavator LUCIE using the Proportional-IntegralPlus (PIP) control strategy. J. Computer-Aided Civil and Infrastruct. Engng, 2004, 12, 16-27.

7 Taylor, C. J., Shaban, E. M., Chotai, A., and Ako, S. Nonlinear control system design for construction robots using state dependent parameter models. In UKACC International Conference (Control 2006), Glasgow, UK, September 2006.

8 Davis, P. F. and Hooper, A. W. Improvement of greenhouse heating control. IEE Proc. Control Theory and Applic., 1991, 138, 249.

9 Bradley, D. A. and Seward, D. W. The development, control and operation of an autonomous robotic excavator. J. Intell. Robotic Syst., 1998, 21, 73-97.

10 Merritt, E. Hydraulic control systems, 1976 (John Wiley, New York).

11 Young, P. C. Stochastic, dynamic modelling and signal processing: time variable and state dependent parameter estimation. In Nonlinear and nonstationary signal processing (Ed. W. J. Fitzgerald), 2000 (Cambridge University Press, Cambridge, UK).

12 McCabe, A. P., Young, P. C., Chotai, A., and Taylor, C. J. Proportional-Integral-Plus (PIP) control of non-linear systems. Syst. Sci., 2000, 26, 25-46.

13 Hebner, A. J., Boon, C. R., and Peugh, G. H. Air patterns and turbulence in an experimental livestock building. J. Agric. Engng Res., 1996, 64, 209-226.

14 Ha, Q., Nguyen, Q., Rye, D., and Durrant-Whyte, H. Impedance control of a hydraulic actuated robotic excavator. J. Automn in Constr., 2000, 9, 421-435.

15 Budny, E., Chlosta, M., and Gutkowski, W. Loadindependent control of a hydraulic excavator. J. Automn in Constr., 2003, 12, 245-254.

16 Taylor, C. J., Pedregal, D. J., Young, P. C., and Tych, W. Environmental time series analysis and forecasting with the Captain Toolbox. Environ. Modelling and Software, 2007, 22(6), 797-814.

17 Young, P. C. Recursive estimation and time series analysis, 1984 (Springer-Verlag, Berlin).

18 Young, P. C. Simplified refined instrumental variable (SRIV) estimation and true digital control (TDC): a tutorial introduction. In Proceedings of 1st European Control Conference, Grenoble, 1991, pp. 1295-1306.
19 Kalman, R. E. A new approach to linear filtering and prediction problems. Trans. ASME, J. Basic Engng, 1960, 83, 95-108.

20 Bryson, A. E. and Ho, Y. C. Applied optimal control, optimization, estimation and control, 1969 (Blaisdell Publishing Company, Waltham, UK).

21 Stables, M. A. and Taylor, C. J. Nonlinear control of ventilation rate using state dependent parameter models. Biosyst. Engng, 2006, 95, 7-18.

22 Burnham, K. J., Dunoyer, A., and Macroft, S. Bilinear controller with PID structure. Computing and Control Engng J., 1999, 10, 63-69.

23 Ziemian, S. J. Bilinear proportional-integral-plus control. PhD Thesis, Coventry University, UK, 2002.

24 Shaban, E. M. Nonlinear control for construction robots using state dependent parameter models. $\mathrm{PhD}$ Thesis, Lancaster University, 2006.

25 Taylor, C. J. Environmental test chamber for the support of learning and teaching in intelligent control. Int. J. Elect. Engng Education, 2004, 41(4), 375-387.

26 Taylor, C. J., Shaban, E. M., Chotai, A., and Ako, S. Development of an automated verticality alignment system for a vibro-lance. In 7th Portuguese International Conference on Automatic Control, Lisbon, Portugal, March 2006.

\section{APPENDIX}

\section{Notation}

$a_{i}\left\{\chi_{k}\right\}$

$A\left(\chi_{k}, \mathrm{z}^{-1}\right) \quad$ denominator polynomial

$b_{\mathrm{b}}\left\{\chi_{k}\right\} \quad$ laboratory excavator boom angle parameter

$b_{\mathrm{d}}\left\{\chi_{k}\right\} \quad$ laboratory excavator dipper angle parameter

$b_{i}\left\{\chi_{k}\right\} \quad$ input state-dependent parameters $(i=1,2, \ldots, m)$

$B\left(\chi_{k}, \mathrm{z}^{-1}\right) \quad$ numerator polynomial

$c$

$C_{i}$ logistic growth function coefficient $\cos \left(\theta_{i}\right)$

$C_{123}$

d $\cos \left(\theta_{1}+\theta_{2}+\theta_{3}\right)$ non-minimal state-space command vector

$f_{i, k} \quad$ feedback coefficients at the $k$ th sample $(i=0, \ldots, n-1)$

$F_{1, k}\left(\mathrm{z}^{-1}\right) \quad$ output feedback polynomial at the $k$ th sample

$\mathbf{F}_{i, k} \quad$ component of the transition matrix at the $k$ th sample $(i=1,2)$

$\mathbf{F}_{k} \quad$ non-minimal state-space transition matrix at the $k$ th sample

$g_{k} \quad$ non-minimal state-space input vector at the $k$ th sample 
$\boldsymbol{h}$ non-minimal state-space observation vector

$k_{\mathrm{I}, k}$

$l_{i}$

$\boldsymbol{l}_{k}$

$\boldsymbol{p}_{k}$

$R_{\mathrm{T}}^{2}$

$S_{i}$

$\mathrm{T}$

$u_{k}$

$u_{\infty}$

$\boldsymbol{U}_{k}$

$\boldsymbol{w}_{k}$

$x_{0}$

$\boldsymbol{x}_{k}$ integral gain at the $k$ th sample

laboratory excavator link lengths

$(i=1,2,3,4)$

state variable feedback gain vector at the $k$ th sample

vector of state-dependent parameters

simulation fit (coefficient of

determination)

$\sin \left(\theta_{i}\right)$

transpose operator

control input at the $k$ th sample

steady-state control input

vector of exogenous variables at the

$k$ th sample

vector of regression variables at the

$k$ th sample

logistic growth function coefficient

non-minimal state vector at the $k$ th

sample $y_{d, k}$

$y_{k}$

$y_{\infty}$

$\mathrm{z}^{-i}$

$z_{k}$

$\alpha_{1}$

$\alpha_{2}$

$\theta$

$\theta_{i}$

$\Theta$

$\tau$

$\phi\{\cdot\}$

$\chi_{k}$ command input at the $k$ th sample control output at the $k$ th sample steady-state output backward shift operator, $\mathrm{z}^{-i} y_{k}=y_{k-i}$ integral-of-error state variable at the $k$ th sample

level coefficient for state-dependent parameter

slope coefficient for state-dependent parameter

logistic growth function coefficient laboratory excavator joint angle $(i=$ $1,2,3,4)$

laboratory excavator bucket

orientation

samples time delay

flexible logistic growth equation state-dependent parameter system variables at the $k$ th sample 